\title{
Correction to: Ceftazidime-Avibactam in Combination with In Vitro Non-susceptible Antimicrobials Versus Ceftazidime-Avibactam in Monotherapy in Critically Ill Patients with Carbapenem-Resistant Klebsiella Pneumoniae Infection: A Retrospective Cohort Study
}

Guanhao Zheng · Jianxin Zhang • Bei Wang · Jiaqi Cai · Lili Wang - Kaixuan Hou •

Yan Zhang $\cdot$ Liang Zhang $\cdot$ Zhitao Yang $\cdot$ Juan He (D) $\cdot$ Xiaolan Bian

Published online: February 28, 2022

(C) The Author(s) 2022

Correction to: Infect Dis Ther (2021)

10:1699-1713 https://doi.org/10.1007/s40121-

021-00479-7

In the original publication of the article, The authors of the following article in Infectious Diseases and Therapy wish to update some of the author details:

https://doi.org/10.1007/s40121-021-00479-7

The original article can be found online at https://doi. org/10.1007/s40121-021-00479-7.

\section{G. Zheng}

Department of Pharmacy, Shanghai Chest Hospital, Shanghai, China

\section{J. Zhang · Y. Zhang}

Department of Pharmacy, Xing'an League People's

Hospital, Ulanhot, China

B. Wang · L. Zhang $(\bowtie)$

Department of Pharmacy, Huashan Hospital Affiliated To Fudan University, Shanghai, China e-mail: cathyzhangliang@126.com

J. Cai

Department of Clinical Laboratory, Kunshan

Hospital Affiliated to Nanjing University of Chinese

Medicine, Kunshan, China

L. Wang

Department of Pharmacy, Wuxi Branch of Ruijin

Hospital, Wuxi, China

K. Hou

Department of Pharmacy, Huaihe Hospital of Henan

University, Kaifeng, China
The corresponding author Bian XiaoLan's work units:

1: Department of Pharmacy, Luwan Branch of Ruijin Hospital Affiliated To Shanghai Jiao Tong University School of Medicine, Shanghai, China;

2: Department of Pharmacy, Ruijin Hospital Affiliated To Shanghai Jiao Tong University School of Medicine, Shanghai, China.

The first author Zheng Guanhao's work units:

\section{Z. Yang $(\bowtie)$ \\ Department of Emergency Intensive Care Unit, Ruijin Hospital Affiliated To Shanghai Jiao Tong University School of Medicine, Shanghai, China e-mail: yangzhitao@hotmail.fr}

\section{G. Zheng · J. He $(\bowtie)$}

Department of Pharmacy, Ruijin Hospital Affiliated

To Shanghai Jiao Tong University School of

Medicine, Shanghai, China

e-mail: hejuanwin@126.com

X. Bian $(\square)$

Department of Pharmacy, Luwan Branch of Ruijin Hospital Affiliated To Shanghai Jiao Tong University School of Medicine, Shanghai, China e-mail: bxl70029@163.com 
1: Department of Pharmacy, Ruijin Hospital Affiliated To Shanghai Jiao Tong University School of Medicine, Shanghai, China;

2: Department of Pharmacy, Shanghai Chest Hospital, Shanghai, China.

The email address for Juan He should be: hejuanwin@126.com

This has been corrected in this paper.

\section{OPEN ACCESS}

This article is licensed under a Creative Commons Attribution-NonCommercial 4.0 International License, which permits any noncommercial use, sharing, adaptation, distribution and reproduction in any medium or format, as long as you give appropriate credit to the original author(s) and the source, provide a link to the Creative Commons licence, and indicate if changes were made. The images or other third party material in this article are included in the article's Creative Commons licence, unless indicated otherwise in a credit line to the material. If material is not included in the article's Creative Commons licence and your intended use is not permitted by statutory regulation or exceeds the permitted use, you will need to obtain permission directly from the copyright holder. To view a copy of this licence, visit http://creativecommons.org/licenses/by$\mathrm{nc} / 4.0 /$. 